ITEP-LAT/2002-14

5 September, 2002

\title{
Geometry of percolating monopole clusters *
}

\author{
P. Yu. Boyko ${ }^{a}$, M.I. Polikarpova , V.I. Zakharov ${ }^{a, b}$ \\ anstitute of Theoretical and Experimental Physics, B.Cheremushkinskaya 25, Moscow, 117259, Russia \\ bMax-Planck Institut für Physik, Föhringer Ring 6, 80805 München, Germany
}

\begin{abstract}
We perform detailed measurements of the geometrical characteristics of the percolating cluster of the magnetic monopole currents in the confining phase of the lattice $S U(2)$ gluodynamics. The Maximal Abelian projection is used to define the monopoles. The use of the geometrical language is motivated by recent observations that the full non-Abelian action associated with the monopoles corresponds to point-like particles on the currently available lattices. Scaling behavior of various quantities is observed.
\end{abstract}

\section{INTRODUCTION}

The monopole condensation is one of the most favored mechanisms of the confinement, for review and references see [1]. Still, there remain important unresolved questions concerning this mechanism. The main point is that the definition of the monopoles, which are Abelian in nature, is not unique within a non-Abelian theory. From pure phenomenological point of view the monopoles defined within the Maximal Abelian Projection (MAP) seem to be most successful.

Monopoles within a particular gauge would not have been of much use if it were not so that they possess remarkable $S U(2)$ invariant properties. In particular, the density of monopoles in the percolating cluster scales as a physical quantity of dimension $d=3$ (see [2] and references therein). It is worth emphasizing that apart from the percolating cluster that fills in the whole of the lattice [3] there exist also finite-size clusters. The percolating cluster is responsible for the confinement and we will concentrate here on its properties.

A further and dramatic evidence for the reality of the MAP monopoles comes from measurements of the full, non-Abelian action associated with the monopoles, see [4] and references therein. Namely, it turned out that at presently available lattices the action is large and ultraviolet divergent:

$S_{\text {mon }}=c_{\text {action }} \cdot(L / a)$,

${ }^{*}$ Talk presented by P.Yu. B. at Lattice 2002, Boston where $c_{\text {action }}$ is a constant, $L$ is the length of the monopole trajectory and $a$ is the lattice spacing. Moreover, the const in Eq. (1) is close to $\ln 7$ which determines the entropy factor. It is well known that the physical mass, entering the propagator is proportional to the difference

$m_{\text {phys }}^{2} \cdot a \approx\left(c_{\text {action }}-\ln 7\right) / a$

The two terms in the r.h.s. of Eq. (2) cancel each other to a great extent and one can say that monopoles are fine-tuned [5].

These observation imply that at presently available lattices the monopoles appear not as extended field configurations but rather as geometrical, point-like objects. Moreover, the monopoles are always observed as (closed) trajectory. Therefore the use of the language of the polymer approach to field theory, or of quantum geometry (see, e.g., [6]) is quite natural.

Below, we present results of measurements of the geometrical characteristics of the monopole trajectories belonging to the percolating cluster. In more detail, the percolating cluster consists of segments of trajectories connecting crossings. We measure, in particular, the average length of the segments and average Euclidean distance between the crossings, the average value of the crossings per unit of 4-volume. In all these cases we observe simple scaling properties which confirm the reality of the MAP monopoles as of physical objects. 
Table 1

$S U(2)$ configurations.

\begin{tabular}{lllllllll}
\hline$\beta$ & 2.30 & 2.35 & 2.40 & 2.40 & 2.45 & 2.50 & 2.55 & 2.60 \\
$L$ & 16 & 16 & 16 & 24 & 24 & 24 & 28 & 28 \\
$N$ & 100 & 100 & 100 & 20 & 10 & 50 & 40 & 50 \\
\hline
\end{tabular}

\section{MEASUREMENTS}

We study the geometry and scaling properties of the monopole trajectories obtained in the maximal Abelian projection of $S U(2)$ lattice gauge theory. To fix the abelian projection we use the simulated annealing algorithm [7]. We study monopoles on symmetric, $L^{4}$, lattices $(16 \leq L \leq$ $24)$ for 7 values of $\beta$. The size of the lattice, $L$, the coupling, $\beta$, and the number of the independent gauge field configurations, $N$, is listed in Table 1 . To fix the physical scale we use $\sqrt{\sigma}=440 \mathrm{Mev}$.

In Section 3 we study the geometrical properties of the monopole trajectories, in Section 4 we discuss the scaling properties of various physical quantities related to monopole trajectories.

\section{LONG-RANGE CORRELATION}

The monopole trajectory is constructed from the links on the dual lattice. It is made from segments (the trajectories between crossings) and crossings of the trajectories. Now we show that the monopole trajectory is not a random walk. Consider the correlation of the direction of the link $C_{1}$ which form the monopole current with the direction of some "initial" link $C_{0}$. The considered link is at the distance $l$ along the monopole trajectory from the initial link. The direction $C_{1}$ can be the same as $C_{0}$, the opposite, or other (neither the same nor the opposite). In our normalization all three correlations are equal to unity for random walk if $l \neq 0$ (for $l=0$ the opposite direction is forbidden). In Fig. 1 we show the correlations of the directions for $\beta=2.6$ on the lattice $28^{4}$.

It is seen that the monopole trajectory has a "long memory". Even for links separated by 17 lattice steps from the initial link the most probable direction is the same as the direction of the initial link.
Figure 1. Correlations of the directions of the links lying on the monopole current.

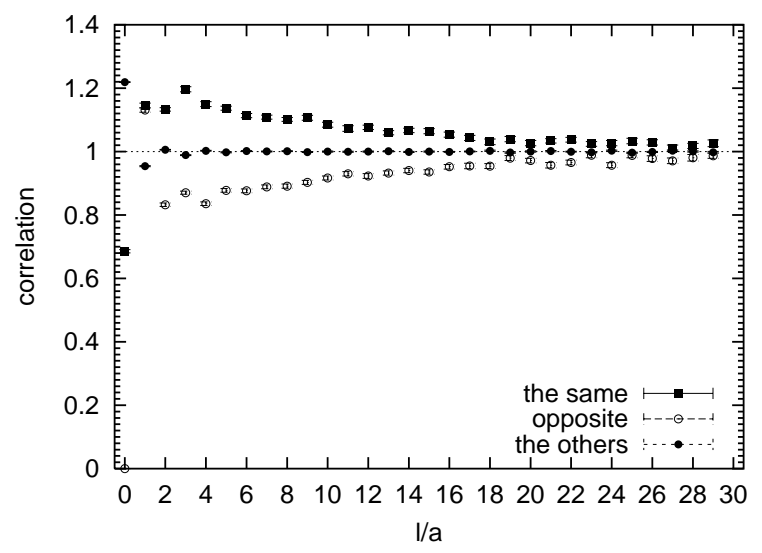

\section{SCALING PROPERTIES}

The average length of the segment of the monopole trajectory between crossings $\langle l\rangle$ scales, see from Fig. 2.

Figure 2. $<l>$ vs. lattice spacing.

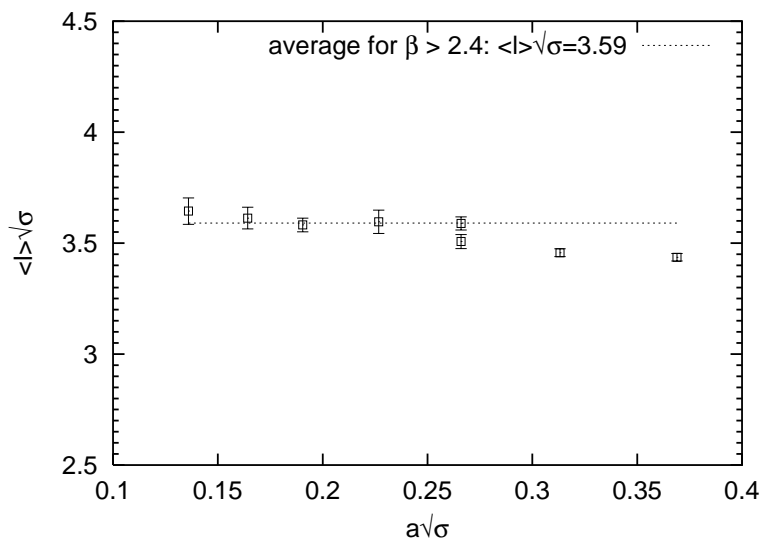


Figure $3 .<d>$ vs. lattice spacing.

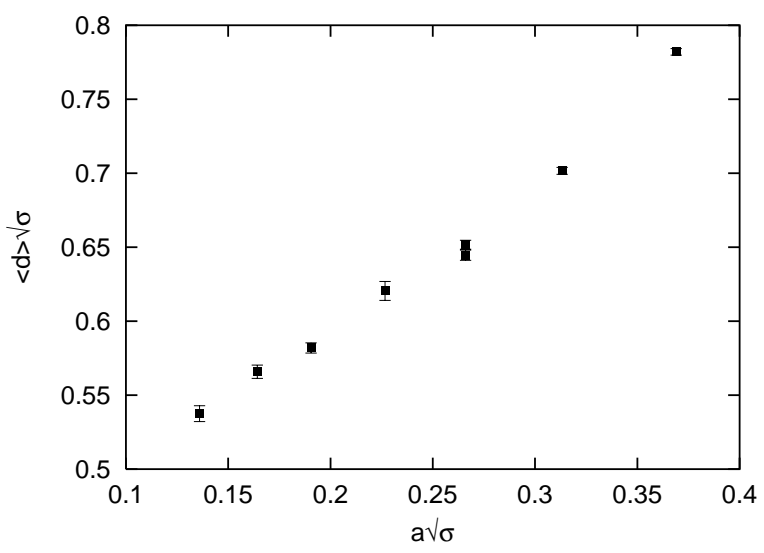

Numerically, $\langle l\rangle \approx 1.6 \mathrm{fm}$ if we average the data for $\beta>2.4$. This scaling behavior confirms strongly the fine tuning of the action and entropy factors, see discussion following Eq. (2).

The data on the average Euclidean distance $<d>$ between crossings are presented in Fig. 3 .

The violations of the scaling are much more substantial in this case. Still they can be approximated by a correction linear in the lattice spacing a.

Figure 4. Number of crossings per unit physical volume vs. lattice spacing.

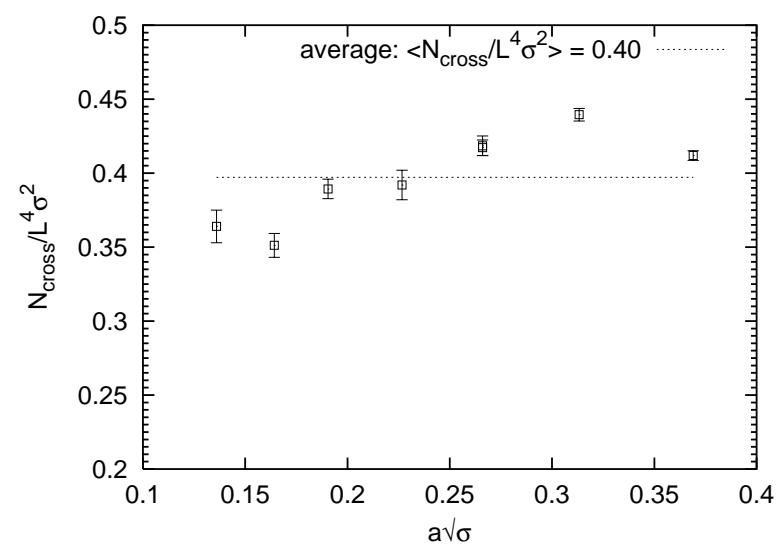

Finally, we found that the number of the crossings of the monopole trajectories per unit physical volume seems to scale as it is seen from Fig. 4 . The average $\left\langle N_{\text {cross }}>/ L^{4} \sigma^{2} \approx 0.4\right.$ corresponds to approximately 10 crossings per hypercube $1 \mathrm{fm}^{4}$. This quantity is probably related to $\phi^{4}$ constant in the effective monopole lagrangian, $\phi$ is the monopole field.

\section{ACKNOWLEDGEMENTS}

The authors are grateful to V. G. Bornyakov, M. N. Chernodub and F. V. Gubarev for useful discussions. M. I. P. is partially supported by grants RFBR 02-02-17308, RFBR 01-02117456, RFBR 00-15-96-786, INTAS-00-00111, and CRDF award RPI-2364-MO-02. P. Yu. B. is partially supported by grants RFBR 02-02-17308 and CDRF award MO-011-0. V.I.Z. is partially supported by grant INTAS-00-00111 and DFG program "From lattice to hadron phenomenology".

\section{REFERENCES}

1. M.N. Chernodub, M.I. Polikarpov, in "Cambridge 1997, Confinement, duality, and nonperturbative aspects of QCD", p. 387; hepth/9710205.

2. V. Bornyakov and M. Muller-Preussker, Nucl. Phys. Proc. Suppl. 106 (2002) 646.

3. T.L. Ivanenko, A.V. Pochinsky, M.I. Polikarpov, Phys. Lett. B 252, 631 (1990);

A. Hart, M. Teper, Phys. Rev. D 58, 014504 (1998).

4. V.G. Bornyakov et al, Phys. Lett. B537 (2002) 291.

5. V.I. Zakharov, hep-ph/0202040.

6. J. Ambjorn, B. Durhuus, Th. Johnsson, "Quantum Geometry ", Cambridge Monographs on Mathematical Physics, (1997).

7. G. S. Bali, V. Bornyakov, M. MullerPreussker and K. Schilling, Phys. Rev. D54 (1996) 2863;

V.G. Bornyakov, D.A. Komarov, M.I. Polikarpov Phys. Lett. B497 (2001) 151. 